

\title{
Article \\ The system of differential equations associated with involutions
}

\author{
Farrukh Dekhkonov ${ }^{1}$
}

Natioanl University of Uzbekistan, University street, 100174, Tashkent, Uzbekistan; f.n.dehqonov@mail.ru

\begin{abstract}
In this paper, we consider with a class of system of differential equations whose argument transforms are involutions. In this an initial value problem for a differential equation with involution is reduced to an initial value problem for a higher order ordinary differential equation. Then either two initial conditions are necessary for a solution; the equation is then reduced to a boundary value problem for a higher order ODE.
\end{abstract}

Keywords: Involution; linear differential equation; fixed point; boundary value problem.

\section{Introduction}

When studying the general properties of functional differential equations, it is always important to find and solve selected classes of equations as explicitly as possible, using methods that are capable of generalization. Differential equations with involutions is one of those classes.

The concept of involution is fundamental for the theory of groups and algebras, but, at the same time, being an object in mathematical analysis properties allow the obtaining of further information concerning this object. In order to be clear in this respect, let us define what we understand by involution in this analytical context. We follow the definitions of [4], [5] and [8].

Definition 1. Let $A \subset \mathbb{R}$ be a set containing more that one point and $f: A \rightarrow A$ a function such that $f$ is not the identity Id. Then $f$ is an involution if

$$
f^{2} \equiv f \circ f=I d
$$

or, equivalently, if

$$
f=f^{-1}
$$

If $A=\mathbb{R}$, we say that $f$ is a strong involution [5].

Example 1. The following involutions are the most common examples:

1. $f: \mathbb{R} \rightarrow \mathbb{R}, f(x)=-x$ is an involution known as reflection.

2. $f: \mathbb{R} \backslash\{0\} \rightarrow \mathbb{R} \backslash\{0\}, f(x)=\frac{1}{x}$ known as inversion.

3. Let $a, b, c \in \mathbb{R}, c b+a^{2} \neq 0, c \neq 0$,

$$
f: \mathbb{R} \backslash\left\{\frac{a}{c}\right\} \rightarrow \mathbb{R} \backslash\left\{\frac{a}{c}\right\}, f(x)=\frac{a x+b}{c x-a},
$$

is a family of functions known as bilinear involutions.

\section{Differential equations with involution}

Differential equations with involutions were introduced for the first time [6] and [7] and since then have become an important part in the general theory of functional differential equations, with applications to certain biomedical models [1], stability of 
motion [2], and the pantograph equation [3]. They can be transformed into ordinary differential equations and thus provide an abundant source of relations with analytic solutions, as well as heuristic ideas for equations of more general nature.

Definition 2. An expression of the form

$$
F\left(x, y\left(f_{1}(x)\right), \ldots, y\left(f_{k}(x)\right), \ldots, y^{(n)}\left(f_{1}(x)\right), \ldots, y^{(n)}\left(f_{k}(x)\right)\right)=0, x \in \mathbb{R},
$$

where $f_{1}, \ldots f_{k}$ are involutions and $F$ is a real function of $n k+1$ real variables is called differential equations with involutions.

Example 2. The solution of the initial-valu problem for the differential equation with reflection of the argument,

$$
y^{\prime}(x)=a y(-x), y(0)=y_{0}
$$

Set a "new" involution function

$$
f(x)=\log _{a}\left(b-a^{x}\right), a>0, a \neq 1, b>0,
$$

where if $a>1, x<\log _{a} b$, if $0<a<1, x>\log _{a} b$.

Consider the following problem

$$
y^{\prime}(x)=y(f(x)), \quad y(0)=y_{0}, f(x)=\ln \left(4-e^{x}\right), x<\ln 4 .
$$

If $y(x)$ is a $C^{1}$ solution then it is $C^{2}$. By differentiation we have

$$
y^{\prime \prime}(x)=f^{\prime}(x) y^{\prime}(f(x)),
$$

than from $f(f(x))=x$ and (1), we get

$$
y^{\prime \prime}(x)=f^{\prime}(x) y(x)=\frac{e^{x}}{e^{x}-4} y(x)
$$

So we have (1) is equivalent to the ordinary Cauchy problem

$$
y^{\prime \prime}(x)=\frac{e^{x}}{e^{x}-4} y(x), y(0)=y_{0}, y^{\prime}(0)=y_{0}, x<\ln 4
$$

Obviously,

$$
y(x)=C_{1}\left(\left(e^{x}-4\right) \ln \left(4-e^{x}\right)-x\left(e^{x}-4\right)+e^{x}\right)+C_{2}\left(e^{x}-4\right) .
$$

Then from the initial conditions, we can write

$$
\left\{\begin{array}{l}
y_{0}=C_{1}(1-3 \ln 3)-3 C_{2} \\
y_{0}=C_{1}(\ln 3+5)+C_{2}
\end{array}\right.
$$

Consequently,

$$
C_{1}=\frac{y_{0}}{4}, C_{2}=-y_{0} \frac{1+\ln 3}{4}
$$

and

$$
y(x)=\frac{y_{0}}{4}\left(\left(e^{x}-4\right) \ln \left(4-e^{x}\right)-x\left(e^{x}-4\right)+e^{x}\right)-\frac{y_{0}(1+\ln 3)}{4}\left(e^{x}-4\right) .
$$

\section{The Main Result and Examples}

In this section, we consider a system of differential equations with involution. 
Theorem 1. Let the initial value problem

$$
\left\{\begin{array}{l}
x^{\prime}(t)=F_{1}(t, x(t), y(t), y(f(t))), \quad x\left(t_{0}\right)=x_{0}, \quad y\left(t_{0}\right)=y_{0}, \\
y^{\prime}(t)=F_{2}(t, x(t), y(t), x(f(t))),
\end{array}\right.
$$

satisfy the following hypotheses:

(1) The function $f(t)$ is a continuously differentiable strong involution with a fixed point $t_{0}$.

(2) The functions $F_{1}, F_{2}$ are defined and are continuously differentiable in the whole space of its arguments.

(3) The given equations are uniquely solvable with respect to $y(f(t)), x(f(t))$ :

$$
\begin{aligned}
& y(f(t))=G_{1}\left(t, x(t), y(t), x^{\prime}(t)\right), \\
& x(f(t))=G_{2}\left(t, x(t), y(t), y^{\prime}(t)\right) .
\end{aligned}
$$

Then the solution of the system of ordinary differential equations

$$
\begin{gathered}
x^{\prime \prime}(t)=\frac{\partial F_{1}}{\partial t}+\frac{\partial F_{1}}{\partial x(t)} x^{\prime}(t)+\frac{\partial F_{1}}{\partial y(t)} y^{\prime}(t)+ \\
+\frac{\partial F_{1}}{\partial x(f(t))} f^{\prime}(t) F_{1}(f(t), x(f(t)), y(f(t)), y(t)),
\end{gathered}
$$

and

$$
\begin{gathered}
y^{\prime \prime}(t)=\frac{\partial F_{2}}{\partial t}+\frac{\partial F_{2}}{\partial x(t)} x^{\prime}(t)+\frac{\partial F_{2}}{\partial y(t)} y^{\prime}(t)+ \\
+\frac{\partial F_{2}}{\partial y(f(t))} f^{\prime}(t) F_{2}(f(t), x(f(t)), y(f(t)), x(t)),
\end{gathered}
$$

(where $y(f(t))$ and $x(f(t))$ are given by expression (4) and (5)) with the initial conditions

$$
x\left(t_{0}\right)=x_{0}, x^{\prime}\left(t_{0}\right)=F_{1}\left(t_{0}, x_{0}, y_{0}, y_{0}\right),
$$

and

$$
y\left(t_{0}\right)=y_{0}, y^{\prime}\left(t_{0}\right)=F_{2}\left(t_{0}, x_{0}, y_{0}, x_{0}\right)
$$

Proof of Theorem 1. Equations (6) and (7) are obtained by differentiating (3). Indeed, we can write

$$
x^{\prime \prime}(t)=\frac{\partial F_{1}}{\partial t}+\frac{\partial F_{1}}{\partial x(t)} x^{\prime}(t)+\frac{\partial F_{1}}{\partial y(t)} y^{\prime}(t)+\frac{\partial F_{1}}{\partial x(f(t))} f^{\prime}(t) x^{\prime}(f(t)),
$$

and

$$
y^{\prime \prime}(t)=\frac{\partial F_{2}}{\partial t}+\frac{\partial F_{2}}{\partial x(t)} x^{\prime}(t)+\frac{\partial F_{2}}{\partial y(t)} y^{\prime}(t)+\frac{\partial F_{2}}{\partial y(f(t))} f^{\prime}(t) y^{\prime}(f(t)),
$$

than from (3) and relation $f(f(t))=t$ its follows that

$$
x^{\prime}(f(t))=F_{1}(f(t), x(f(t)), y(f(t)), y(t)),
$$

and

$$
y^{\prime}(f(t))=F_{2}(f(t), x(f(t)), y(f(t)), x(t)) .
$$

The second of the initial conditions (8), (9) are compatibility condition and is found from (3), with regard to (3) initial condition and $f\left(t_{0}\right)=t_{0}$. 
Example 3. We consider the following initial value problem

$$
\left\{\begin{array}{l}
x^{\prime}(t)=y(f(t)), \\
y^{\prime}(t)=x(f(t)),
\end{array} \quad x(0)=x_{0}, \quad y(0)=y_{0}, \quad f(t)=-t .\right.
$$

We can write

$$
\left\{\begin{array}{l}
x^{\prime \prime}(t)=f^{\prime}(t) y^{\prime}(f(t)), \\
y^{\prime \prime}(t)=f^{\prime}(t) x^{\prime}(f(t)) .
\end{array}\right.
$$

Than from $f(f(t))=t$ and (10) we get

$$
\left\{\begin{array}{l}
x^{\prime \prime}(t)=f^{\prime}(t) x(t) \\
y^{\prime \prime}(t)=f^{\prime}(t) y(t)
\end{array}\right.
$$

So we have (10) is equivalent to the boundary value problem

$$
\left\{\begin{array}{l}
x^{\prime \prime}(t)+x(t)=0, \\
y^{\prime \prime}(t)+y(t)=0,
\end{array} \quad x(0)=x_{0}, \quad y(0)=y_{0}, \quad x^{\prime}(0)=y_{0}, \quad y^{\prime}(0)=x_{0}\right.
$$

Obviously,

$$
\left\{\begin{array}{l}
x(t)=C_{1} \cos t+C_{2} \sin t \\
y(t)=C_{3} \cos t+C_{4} \sin t
\end{array}\right.
$$

Then from the boundary conditions, we can write

$$
\left\{\begin{array}{l}
x(t)=x_{0} \cos t+y_{0} \sin t \\
y(t)=y_{0} \cos t+x_{0} \sin t
\end{array}\right.
$$

Example 4. We consider the following problem

$$
\left\{\begin{array}{l}
x^{\prime}(t)=y(f(t)), \\
y^{\prime}(t)=x(f(t)),
\end{array} \quad x\left(\frac{\pi}{2}\right)=x_{0}, \quad y\left(\frac{\pi}{2}\right)=y_{0}, \quad f(t)=\pi-t, \quad a \in \mathbb{R} .\right.
$$

We can write

$$
\left\{\begin{array}{l}
x^{\prime \prime}(t)=f^{\prime}(t) y^{\prime}(f(t)), \\
y^{\prime \prime}(t)=f^{\prime}(t) x^{\prime}(f(t)) .
\end{array}\right.
$$

Than from $f(f(t))=t$ and given system of differential equation we get

$$
\left\{\begin{array}{l}
x^{\prime \prime}(t)=f^{\prime}(t) x(t) \\
y^{\prime \prime}(t)=f^{\prime}(t) y(t)
\end{array}\right.
$$

So we have given system of differential equation is equivalent to the boundary value problem

$$
\left\{\begin{array}{l}
x^{\prime \prime}(t)+x(t)=0, \\
y^{\prime \prime}(t)+y(t)=0,
\end{array} \quad x\left(\frac{\pi}{2}\right)=x_{0}, \quad y\left(\frac{\pi}{2}\right)=y_{0}, \quad x^{\prime}\left(\frac{\pi}{2}\right)=y_{0}, \quad y^{\prime}\left(\frac{\pi}{2}\right)=x_{0}\right.
$$

Obviously,

$$
\left\{\begin{array}{l}
x(t)=C_{1} \cos t+C_{2} \sin t \\
y(t)=C_{3} \cos t+C_{4} \sin t
\end{array}\right.
$$


Then from the boundary conditions, we can write

$$
\left\{\begin{array}{l}
x(t)=-y_{0} \cos t+x_{0} \sin t \\
y(t)=-x_{0} \cos t+y_{0} \sin t
\end{array}\right.
$$

Theorem 2. Let the initial value problem

$$
\left\{\begin{array}{l}
x^{\prime}(t)=F_{1}(t, x(t), y(t), x(f(t))), \\
y^{\prime}(t)=F_{2}(t, x(t), y(t), y(f(t))),
\end{array} \quad x\left(t_{0}\right)=x_{0}, y\left(t_{0}\right)=y_{0},\right.
$$

satisfy the following hypotheses:

(1) The function $f(t)$ is a continuously differentiable strong involution with a fixed point $t_{0}$.

(2) The functions $F_{1}, F_{2}$ are defined and are continuously differentiable in the whole space of its arguments.

(3) The given equations are uniquely solvable with respect to $y(f(t)), x(f(t))$ :

$$
\begin{aligned}
& x(f(t))=G_{1}\left(t, x(t), y(t), x^{\prime}(t)\right), \\
& y(f(t))=G_{2}\left(t, x(t), y(t), y^{\prime}(t)\right) .
\end{aligned}
$$

Then the solution of the system of ordinary differential equations

$$
\begin{gathered}
x^{\prime \prime}(t)=\frac{\partial F_{1}}{\partial t}+\frac{\partial F_{1}}{\partial x(t)} x^{\prime}(t)+\frac{\partial F_{1}}{\partial y(t)} y^{\prime}(t)+ \\
+\frac{\partial F_{1}}{\partial y(f(t))} f^{\prime}(t) F_{1}(f(t), x(f(t)), y(f(t)), x(t)),
\end{gathered}
$$

and

$$
\begin{gathered}
y^{\prime \prime}(t)=\frac{\partial F_{2}}{\partial t}+\frac{\partial F_{2}}{\partial x(t)} x^{\prime}(t)+\frac{\partial F_{2}}{\partial y(t)} y^{\prime}(t)+ \\
+\frac{\partial F_{2}}{\partial x(f(t))} f^{\prime}(t) F_{2}(f(t), x(f(t)), y(f(t)), y(t))
\end{gathered}
$$

(where $x(f(t))$ and $y(f(t))$ are given by expression (12) and (13)) with the initial conditions

$$
x\left(t_{0}\right)=x_{0}, x^{\prime}\left(t_{0}\right)=F_{1}\left(t_{0}, x_{0}, y_{0}, x_{0}\right),
$$

and

$$
y\left(t_{0}\right)=y_{0}, y^{\prime}\left(t_{0}\right)=F_{2}\left(t_{0}, x_{0}, y_{0}, y_{0}\right)
$$

Proof of Theorem 2. The proof of theorem 2 comes from theorem 1.

Example 5. We consider the following problem

$$
\left\{\begin{array}{l}
x^{\prime}(t)=\alpha x(f(t)), \\
y^{\prime}(t)=\beta y(f(t)),
\end{array} \quad x\left(t_{0}\right)=x_{0}, \quad y\left(t_{0}\right)=y_{0}, \quad f(t)=\frac{1}{t}, \quad \alpha, \beta=\right.\text { const. }
$$

\section{References}

1. Busenberg S.; Travis C. On the Use of Reducible-Functional Differential Equations in Biological Modeles. J. Math. Anal. Appl 1982, 89, 46-66.

2. Castelan, W. G.; Infante, E.F. On a Functional Equation Arising in the Stability Theory of Difference-Differential Equations Quart. Appl. Math 1977, 35, 311-319.

3. Derfel, G.; Iserles C. The Pantograph Equation in the Complex Plane J. Math. Anal. Appl 1997, $213,117-132$. 
4. Wiener, J.; Watkins, W. A glimpse into the wonderland of involutions Missouri J.Math.Sci 2002, 14(3), 175-185.

5. Wiener, J. Generalized solutions of functional differential equations World Scientific 1993.

6. Wiener, J. Differential equations with Involutions Differential Equations 1969, 5, 1131-1137.

7. Wiener, J. Differential equations in Partial Derivatives with Involutions Differential Equations 1970, 6, 1320-1322.

8. Watkins, W. Modified Wiener equations Int. J. Math. Sci 2001, 27, 347-356. 
